\title{
Therapeutic effect of the immunomodulatory drug lenalidomide, but not pomalidomide, in experimental models of rheumatoid arthritis and inflammatory bowel disease
}

\author{
Belen Lopez-Millan ${ }^{1}$, Rafael Diaz de la Guardia ${ }^{1}$, Heleia Roca-Ho ${ }^{1}$, Carmen M García-Herrero ${ }^{2}$, \\ Jessie R Lavoie ${ }^{3}$, Michael Rosu-Myles ${ }^{3}$, Elena Gonzalez-Rey ${ }^{4}$, Francisco O'Valle ${ }^{5}$, Gabriel Criado ${ }^{2}$, \\ Mario Delgado ${ }^{4}$ and Pablo Menendez ${ }^{1,6}$
}

Thalidomide is an immunomodulatory drug (IMiD) with proven therapeutic action in several autoimmune/inflammatory diseases; however, its inherent high toxicity has led to the development of more powerful and safer thalidomide analogs, including lenalidomide and pomalidomide. These are new generation IMiDs that exhibit direct antitumor activity as well as anti-inflammatory/immunomodulatory properties, and are FDA-approved for the treatment of several hematological malignances. Here we investigated the potential therapeutic effects of lenalidomide and pomalidomide in several experimental murine models of autoimmune/inflammatory diseases: 2,4,6-trinitrobenzene sulfonic acid- and dextran sulfate sodium-induced inflammatory bowel disease and type II collagen-induced arthritis. Lenalidomide displayed a strong therapeutic effect in all these models of autoimmune/inflammatory diseases, while the effect of pomalidomide was less pronounced. In vitro experiments confirmed the immunosuppressive effect of both IMiDs on the proliferative response of stimulated human lymphocytes and on the balance of secreted cytokines toward an anti-inflammatory profile. We conclude that lenalidomide may offer a therapeutic opportunity against autoimmune/inflammatory diseases.

Experimental \& Molecular Medicine (2017) 49, e290; doi:10.1038/emm.2016.143; published online 3 February 2017

\section{INTRODUCTION}

Autoimmune and inflammatory diseases constitute a variety of debilitating disorders in which inflammation is the hallmark of the disease. ${ }^{1}$ Immune-mediated inflammatory conditions of both the gastrointestinal tract, such as Crohn's disease and ulcerative colitis (collectively known as inflammatory bowel disease (IBD)), and the synovial tissue in joints, such as rheumatoid arthritis (RA), are prevalent organ/tissue-specific inflammatory diseases that have benefited from preclinical investigation in animal models, resulting in a significant improvement outcome for patients. ${ }^{2-4}$ The primary goal of any treatment is to control pain and inflammation; however, currently available therapies for inflammatory diseases are based on immunosuppressive agents (monoclonal antibodies, steroids and biological drugs) that are not entirely effective and are often nonspecific, ${ }^{5,6}$ illustrating the need for novel therapeutic approaches.

The immunomodulatory drugs (IMiDs) are a group of therapeutic analogs of thalidomide, a synthetic glutamic acid derivative with beneficial effects in a variety of inflammatory

\footnotetext{
${ }^{1}$ Department of Biomedicine, School of Medicine, Josep Carreras Leukemia Research Institute, University of Barcelona, Barcelona, Spain; ${ }^{2}$ Inflammatory and Autoimmune Diseases Group, Hospital 12 de Octubre Research Institute, Madrid, Spain; ${ }^{3}$ Centre for Biologics Evaluation, Biologics and Genetic Therapies Directorate, Health Products and Food Branch, Health Canada, Ontario, Ottawa, Canada; 'Instituto de Parasitología y Biomedicina 'López Neyra', Armilla, Granada, Spain; ${ }^{5}$ Department of Pathology, School of Medicine, University of Granada, Granada, Spain and ${ }^{6}$ Instituciò Catalana Recerca i Estudis Avançats (ICREA), Pg Lluís Companys 23, Barcelona, Spain

Correspondence: Dr G Criado, Inflammatory and Autoimmune Diseases Group, Hospital 12 de Octubre Research Institute, Avda de Córdoba s/n, 28041 Madrid, Spain.

E-mail: gcriado@h12o.es

or Dr M Delgado, Instituto de Parasitología y Biomedicina 'López Neyra', Avda del Conocimiento s/n., 18016 Armilla, Granada, Spain.

E-mail: mdelgado@ipbln.csic.es

or Professor P Menendez, Department of Biomedicine, School of Medicine, Josep Carreras Leukemia Research Institute, University of Barcelona, Casanova 143, 08036 Barcelona, Spain.

E-mail: pmenendez@carrerasresearch.org

Received 14 July 2016; revised 5 October 2016; accepted 10 October 2016
} 
disorders. $^{7-9}$ Despite its anti-inflammatory properties, thalidomide is associated with high toxicity and congenital abnormalities, ${ }^{10}$ and was withdrawn from the market in the early 1960s. ${ }^{11}$ Currently, it is FDA-approved solely for the treatment of erythema nodosumleprosum. ${ }^{11}$ IMiDs have emerged as potent and safe antineoplastic agents with immunomodulatory and antiangiogenic activities. ${ }^{12-14}$ The two first-in-class IMiDs with a favorable toxicity profile and increased efficacy are lenalidomide and pomalidomide, ${ }^{7,12}$ which are first-line treatments in several hematological malignancies including multiple myeloma and myelodysplastic syndromes. ${ }^{14,15}$ The postulated mechanisms of action for IMiDs include blocking signaling through nuclear factorkappa B (NFkB), downregulation of TNF $\alpha$ and cyclooxygenase 2 (COX-2), and T-cell co-stimulatory properties, abolishing the requirement for secondary co-stimulation signals from antigen-presenting cells. ${ }^{16-18}$

Given their immunomodulatory and anti-inflammatory properties, IMiDs may have a therapeutic window not only in neoplasia but also in diseases characterized by an inflammatory phenotype. Here we aimed at investigating the potential therapeutic efficacy of lenalidomide and pomalidomide in wellestablished experimental murine models of IBD and RA. ${ }^{19-22}$ We found that lenalidomide produced a strong therapeutic effect in three different models of inflammatory disease, whereas pomalidomide treatment resulted in a lesspronounced amelioration of symptoms. In vitro experiments confirmed an immunosuppressive effect of both IMiDs on the proliferation of stimulated human and on the balance of secreted cytokines towards an anti-inflammatory profile. Together, these findings support the use of IMiDs as a therapeutic option for both IBD and RA.

\section{MATERIALS AND METHODS}

\section{Induction and treatment of experimental IBD}

To induce IBD in vivo, $3 \mathrm{mg}$ of 2,4,6-trinitrobenzenesulfonic acid (TNBS, Sigma, St Quentin-Fallavier, France) in 50\% ethanol $(100 \mu \mathrm{l})$ was administered intrarectally in 7-week-old Bagg Albino/c male mice. Control mice received $50 \%$ ethanol alone. Animals ( $n=10$ per group) were treated intraperitoneally (i.p.) with vehicle (PBS-1\% DMSO, controls) or with lenalidomide $\left(50 \mathrm{mg} \mathrm{kg}^{-1}\right.$ per day) or pomalidomide ( $50 \mathrm{mg} \mathrm{kg}^{-1}$ per day), for 3 days starting $12 \mathrm{~h}$ after TNBS instillation. Animals were monitored daily for the appearance of diarrhea, body weight loss and survival. Colons (from the cecum to the anus) were removed immediately after death or at day 7 , and were evaluated for macroscopic damage (graded on a scale $0-10$ ) based on criteria reflecting inflammation (hyperemia, bowel thickening and ulceration extent) by two independent researchers in a blinded manner: ulceration $(0=$ normal appearance, $1=$ focal hyperemia, no ulcers, $2=$ ulceration without hyperemia or bowel wall thickening, $3=$ ulceration with inflammation at one site, $4=$ two or more sites of ulceration and inflammation, $5=$ major sites of damage extending $>1 \mathrm{~cm}$ along length of colon, $6-10=$ when an area of damage extended $>2 \mathrm{~cm}$ along length of colon, score is increased by 1 for each additional $\mathrm{cm}$ of involvement). Scores for stool consistency and rectal bleeding were assessed by two blinded observers as described: ${ }^{19,23} 0=$ normal stool appearance; $1=$ slight decrease in stool consistency; 2=moderate decrease in stool consistency; $3=$ moderate decrease in stool consistency and presence of blood in stool; $4=$ severe watery diarrhea and moderate/severe bleeding in stool. For histopathologic analysis, colon was fixed in $10 \%$ buffered formalin phosphate, paraffin embedded and sectioned. Sections were stained with hematoxylin-eosin, and inflammation was graded from 0 to 4 by an independent pathologist as described. ${ }^{19,24} 0=$ no signs of inflammation; $1=$ low leukocyte infiltration; $2=$ moderate leukocyte infiltration; $3=$ high leukocyte infiltration, moderate fibrosis, high vascular density, thickening of the colon wall, moderate goblet cell loss and focal loss of crypts; and $4=$ transmural infiltrations, massive loss of goblet cell, extensive fibrosis and diffuse loss of crypts. The animal care committee of the IPBLN-CSIC approved all procedures.

Alternatively, acute colitis was induced in male $\mathrm{C} 57 \mathrm{Bl} / 6$ mice (7-week old, Charles River) by administering 3\% dextran sulfate sodium (DSS; molecular weight 20,000 Da; Sigma) from day 0 to day 7 in the drinking water ad libitum. Animals hydrated with normal water were used as naive controls. At days 3, 4 and 5, vehicle, lenalidomide ( $50 \mathrm{mg} \mathrm{kg}^{-1}$ per day) or pomalidomide $\left(50 \mathrm{mg} \mathrm{kg}^{-1}\right.$ per day) were injected i.p. in the DSS-treated animals. Colitis severity was assessed daily by scoring $(0-4)$ the clinical disease activity by evaluating stool consistency, presence of fecal blood and weight loss: weight loss $(0=0 \%, 0.5=1-10 \%, 1=11-15 \%, 1.5=16-20 \%, 2 \geqslant 20 \%)$, diarrhea $(0=$ normal stool, $0.5=$ soft stool and minimal wet anal fur $/$ tail, $1=$ diarrhea and moderate-to-severe wet anal fur/tail) and frank rectal bleeding $(0=$ absent, $0.5=$ present but minimal, $1=$ moderate/severe $)$. At sacrifice, the entire colon was removed from the cecum to the anus, and colon length and weight were measured as indirect inflammation markers. The macroscopic colonic damage score (scale 0-8) was assessed based on the grade of tissue adhesion, presence of ulceration and wall thickness: ulceration $(0=$ normal appearance, $1=$ focal hyperaemia, no ulcers, $2=$ ulceration without hyperemia or bowel wall thickening, $3=$ ulceration with inflammation at one site, $4=$ two or more sites of ulceration and inflammation, $5=$ major sites of damage extending $>1 \mathrm{~cm}$ along length of colon), adhesions $(0=$ no adhesions, $1=$ minor adhesions, colon can be easily separated from the other tissues, $2=$ major adhesions) and thickness (maximal bowel wall thickness, in mm, measured with a calliper).

\section{Induction and treatment of collagen-induced arthritis}

DBA/1OlaHsd mice were purchased from Harlan Laboratories and housed under specific pathogen-free conditions. Ten-week-old male mice were immunized by intradermal injection at the base of the tail with $200 \mu \mathrm{g}$ of chicken CII in complete Freund's adjuvant as described..$^{20}$ Lenalidomide and pomalidomide $\left(50 \mathrm{mg} \mathrm{kg}^{-1}\right)$ or PBS-1\% DMSO (vehicle, control mice) were administered daily by i.p. injection starting on the day of arthritis onset. Arthritis severity was graded daily for each paw by two independent observers using the following scoring system: $0=$ normal, $1=$ slight swelling and erythema, $2=$ pronounced edematous swelling and $3=$ stiffness of the joint. This yielded a maximum score of 12 per mouse. Disease severity was expressed as mean clinical score \pm s.e.m. per group of treatment. Sixteen mice per group were included, eight in each experiment. All animal procedures were approved by the Hospital 12 de Octubre Animal Welfare Committee.

\section{Serum measurements}

Serum was collected from mice with collagen-induced arthritis (CIA) after 10 days of treatment. Serum anti-CII-specific antibodies IgM, 
total IgG, IgG1 and IgG2a subclasses were determined by ELISA in 96-well ELISA plates coated with CII $\left(2 \mu \mathrm{g} \mathrm{ml}^{-1}\right.$ in Tris buffer $(0.05 \mathrm{M}$ Tris, $0.2 \mathrm{M} \mathrm{NaCl}, \mathrm{pH} 7.4$ ) and horseradish peroxidase-coupled goat anti-mouse Ig class-specific antibodies. Relative antibody units (RU) were calculated as the $\log 10$ of the reciprocal of the dilution giving
$50 \%$ of maximal optical density. Serum IL6 levels were assessed by ELISA (Mouse IL-6 ELISA MAX Standard; BioLegend, San Diego, CA, USA). Serum levels of IL1 $\alpha$, IL17, IL13, IL4, IL27 were assessed by FlowCytomix Multiple Analyte Detection System (Mouse 13plex kit FlowCytomix, eBioscience, Vienna, Austria).
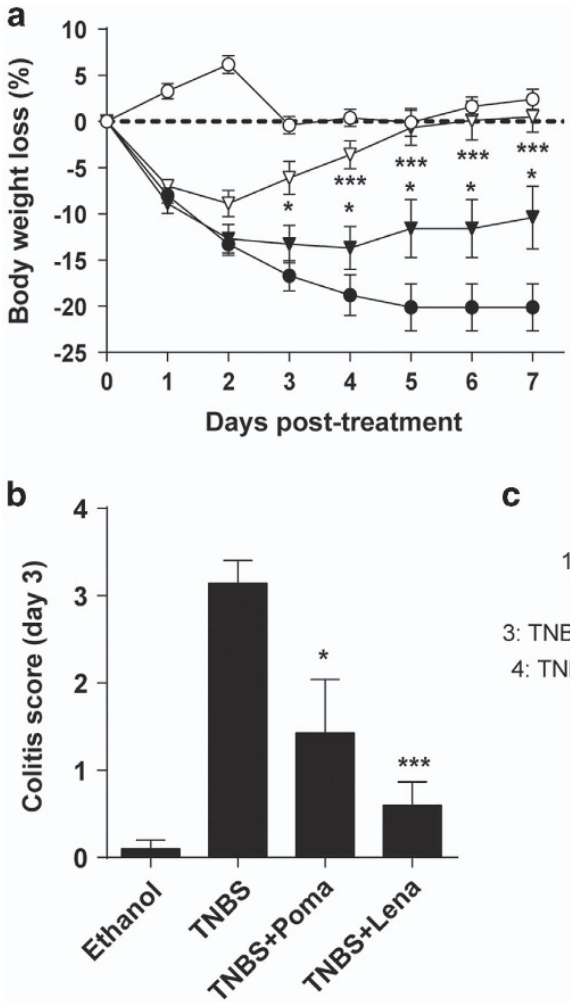

d

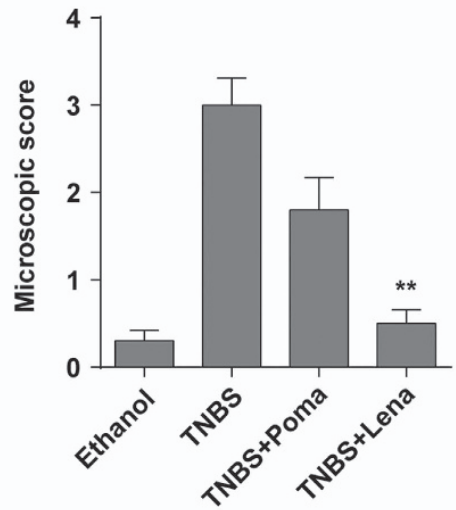

C

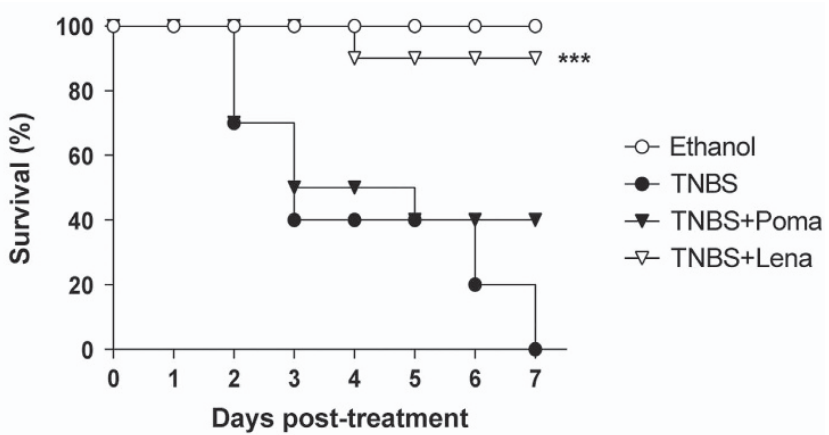

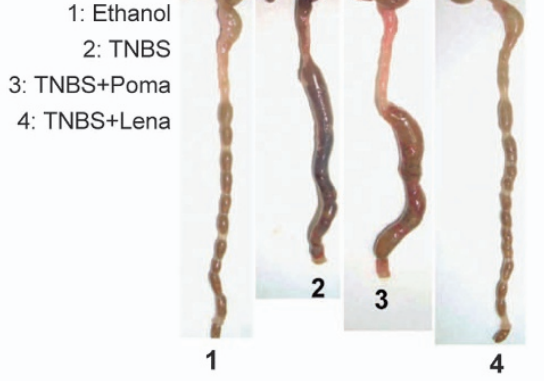

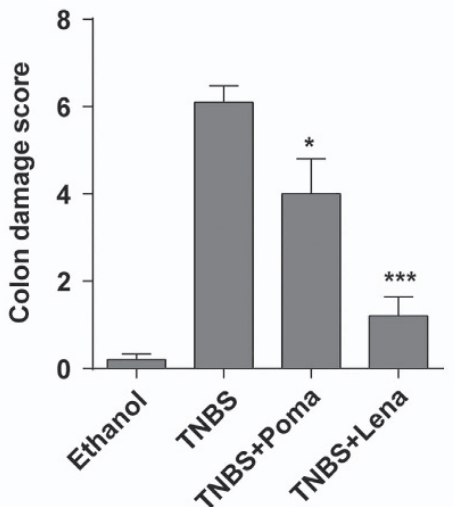

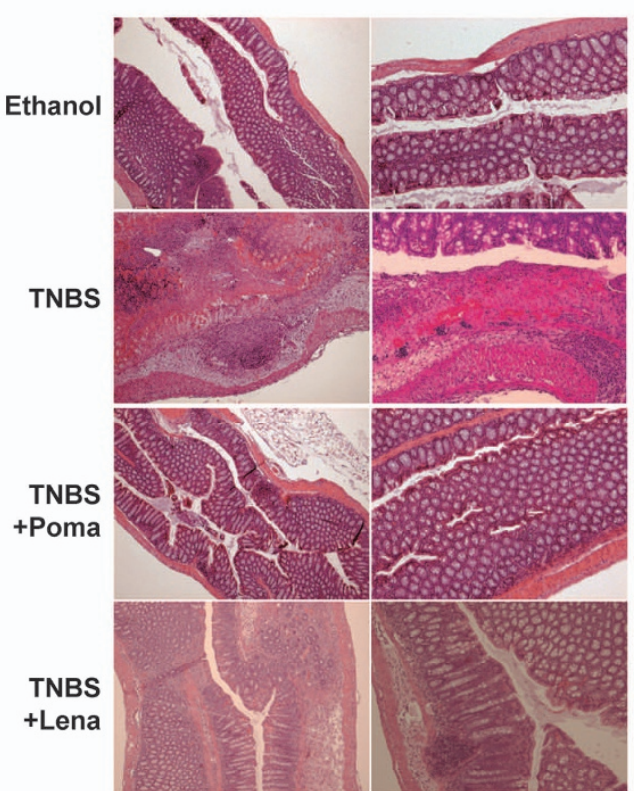

Figure 1 Lenalidomide treatment protects against 2,4,6-trinitrobenzene sulfonic acid (TNBS)-induced acute severe colitis. Colitis was induced by intracolonic administration of TNBS and mice were treated i.p. with lenalidomide or pomalidomide for 3 days, starting at $12 \mathrm{~h}$ after TNBS injection. Clinical evolution was monitored by determining the daily body weight loss and survival (a) as well as measuring the colitis score (at day 3) (b), the macroscopic colonic damage score (c) and the histopathologic score (at day 3) (d). Data are expressed as mean \pm s.e.m.; $n=10$ mice per group in a-c; $n=5$ mice per group in d. Statistical differences between groups were calculated as described in Materials and methods. ${ }^{*} P<0.05 ; * * * P<0.01 ; * * * P<0.001$. 


\section{Lymphocyte proliferation and cytokine production}

Peripheral blood mononuclear cells (PBMCs) were isolated from buffy coats from healthy volunteers by Ficoll-Hypaque gradients. Samples designated for the determination of proliferation were labeled with $5 \mu \mathrm{m}$ carboxyfluorescein diacetate succinimidyl ester (CSFE) (Invitrogen, Eugene, OR, USA) before culture. CSFE-labeled PBMCs $\left(1.2 \times 10^{5}\right)$ were placed in 96-well flat-bottom plates in $200 \mu \mathrm{l}$ of culture medium (DMEM supplemented with $10 \%$ FBS, $20 \mathrm{~mm} \mathrm{~L}$ glutamine, and $1 \%$ penicillin and streptomycin) per well and cultured for 5 days $\left(37^{\circ} \mathrm{C}, 5 \% \mathrm{CO}_{2}\right)$ with or without $10 \mu \mathrm{g} \mathrm{ml} \mathrm{l}^{-1}$ phytohemaglutinin (PHA) (Sigma-Aldrich). PHA-stimulated PBMCs were also treated or not with $10 \mu \mathrm{m}$ of lenalidomide or pomalidomide for 5 days. The number of cycling cells was determined by flow cytometry and was calculated as a percentage of $\mathrm{CSFE}^{\text {mild/low }}$ cells that had divided, multiplied by the total number of cells. ${ }^{21,22}$ To determine cytokine production, PBMCs $\left(1.2 \times 10^{5}\right)$ were plated in 96-well flatbottom plates in $200 \mu \mathrm{l}$ of culture medium and stimulated or not with $10 \mu \mathrm{g} \mathrm{ml}^{-1}$ PHA for 5 days or $0.1 \mu \mathrm{g} \mathrm{m}{ }^{-1}$ lipopolysaccharide (SigmaAldrich) for $7 \mathrm{~h}$. PBMCs were also treated or not with $10 \mu \mathrm{m}$ lenalidomide or pomalidomide during the stimulation period. Culture supernatants were collected at the end of treatment and analyzed by
Luminex Multiplex assays. Interferon gamma (IFNy), IL17 and IL13 were determined in PHA-stimulated PMBCs while the levels of the other cytokines were determined in lipopolysaccharide-stimulated PBMCs. Cytokine concentration was normalized to 1. Thus, relative units were calculated as the fold-change of stimulated PBMC (with or without IMiD treatment) relative to non-stimulated PBMCs.

\section{Statistical analysis}

Descriptive analysis of each experiment was performed to compare the severity of the disease between experimental groups. The impact of lenaledomide and pomaledomide on the scores and the course of disease was analyzed by two-way analysis of variance for repeated measures. Differences in antibody levels and mouse interleukins between lenalidomide and control groups were compared using the Mann-Whitney $U$-test for two independent samples. Differences in cytokine levels and proliferation between the IMiD group and the non-treated group were compared using an independent sample $t$-test. Analysis was performed using GraphPad Prism software (version 6.0, GraphPad; La Jolla, CA, USA). $P$-values below 0.05 were considered statistically significant. a

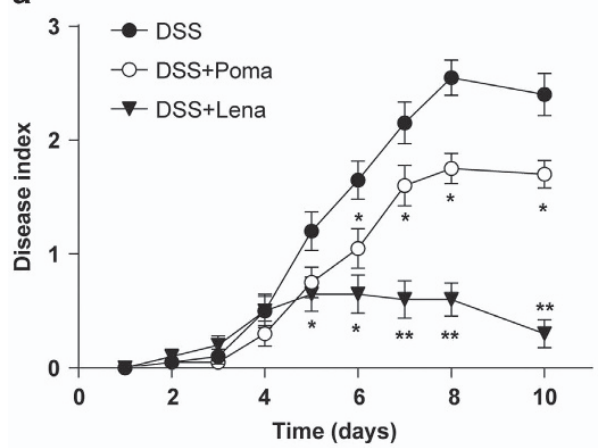

b

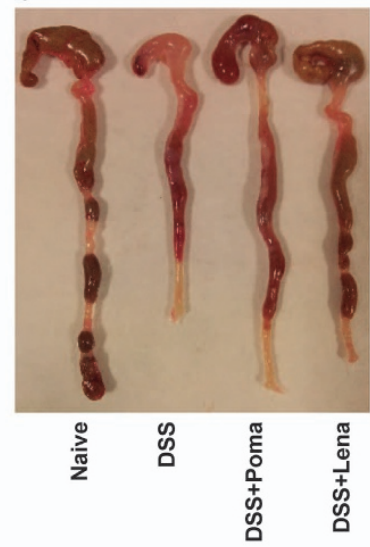

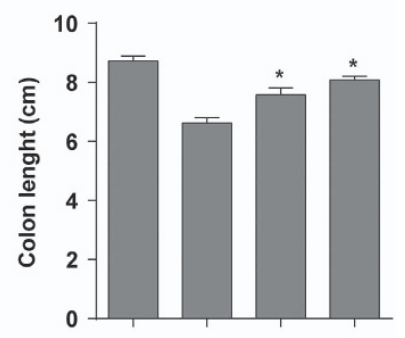
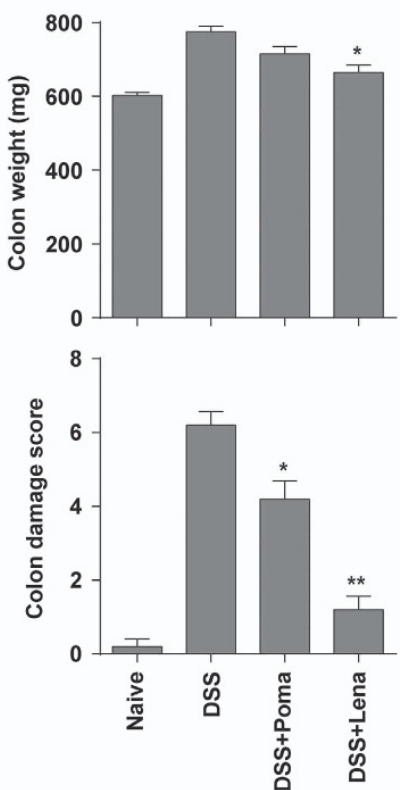

Figure 2 Lenalidomide treatment protects from dextran sulfate sodium (DSS)-induced acute colitis. Mice received 3\% DSS in the drinking water from day 0 to day 7. Lenalidomide or pomalidomide were administered intraperitoneally once a day for 3 days, starting at day 3 . (a) Disease activity scores were determined daily. (b) Colon damage scores and colon length and weight were determined at day 8. Mice receiving tap water instead of DSS were used as controls (naive). Data are expressed as mean \pm s.e.m.; $n=10$ mice per group in a; $n=5$ mice per group in $\mathbf{b}$. Statistical differences between groups were calculated as described in Materials and methods. ${ }^{*} P<0.05 ;{ }^{* *} P<0.01$. 

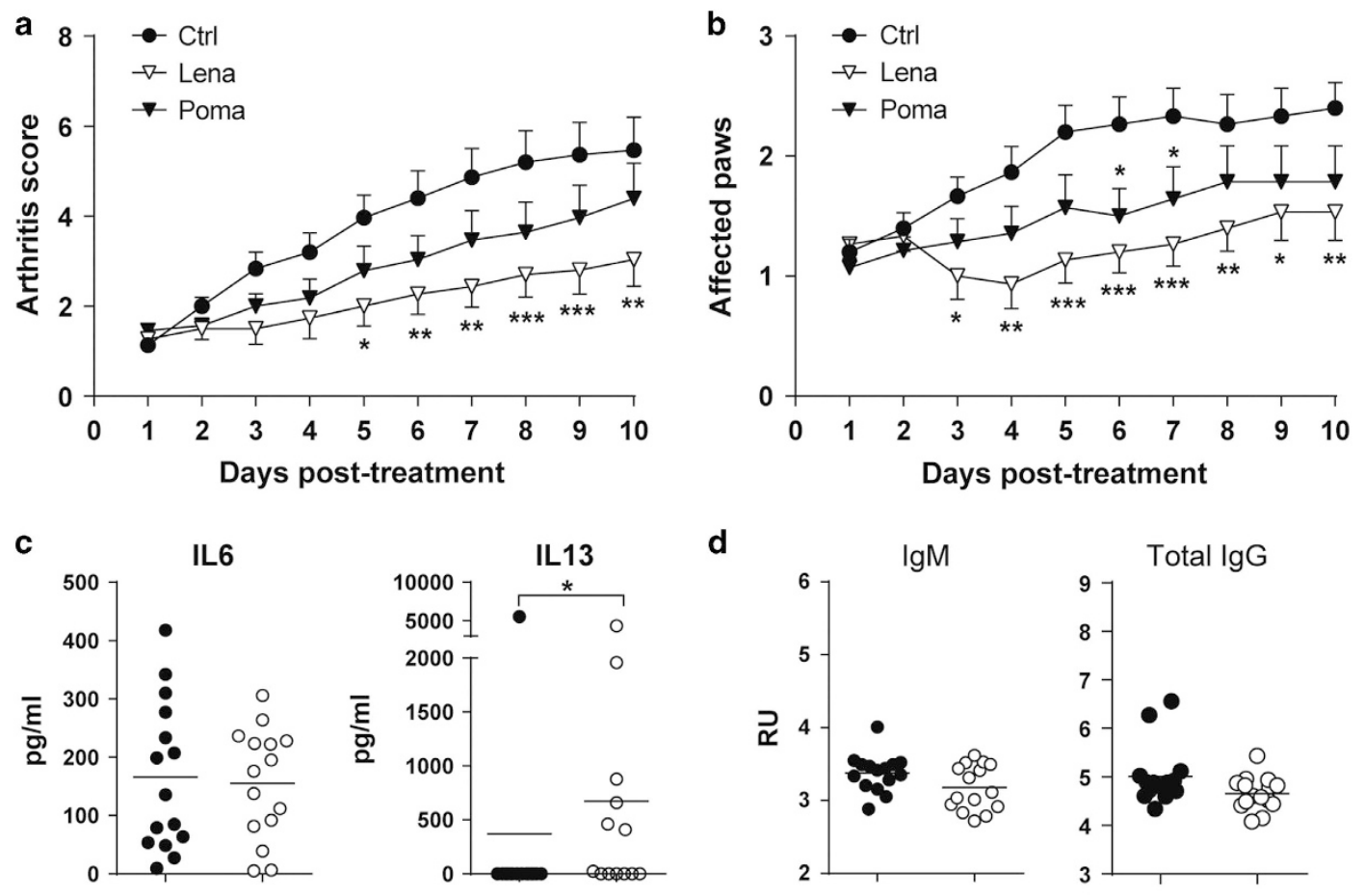

d
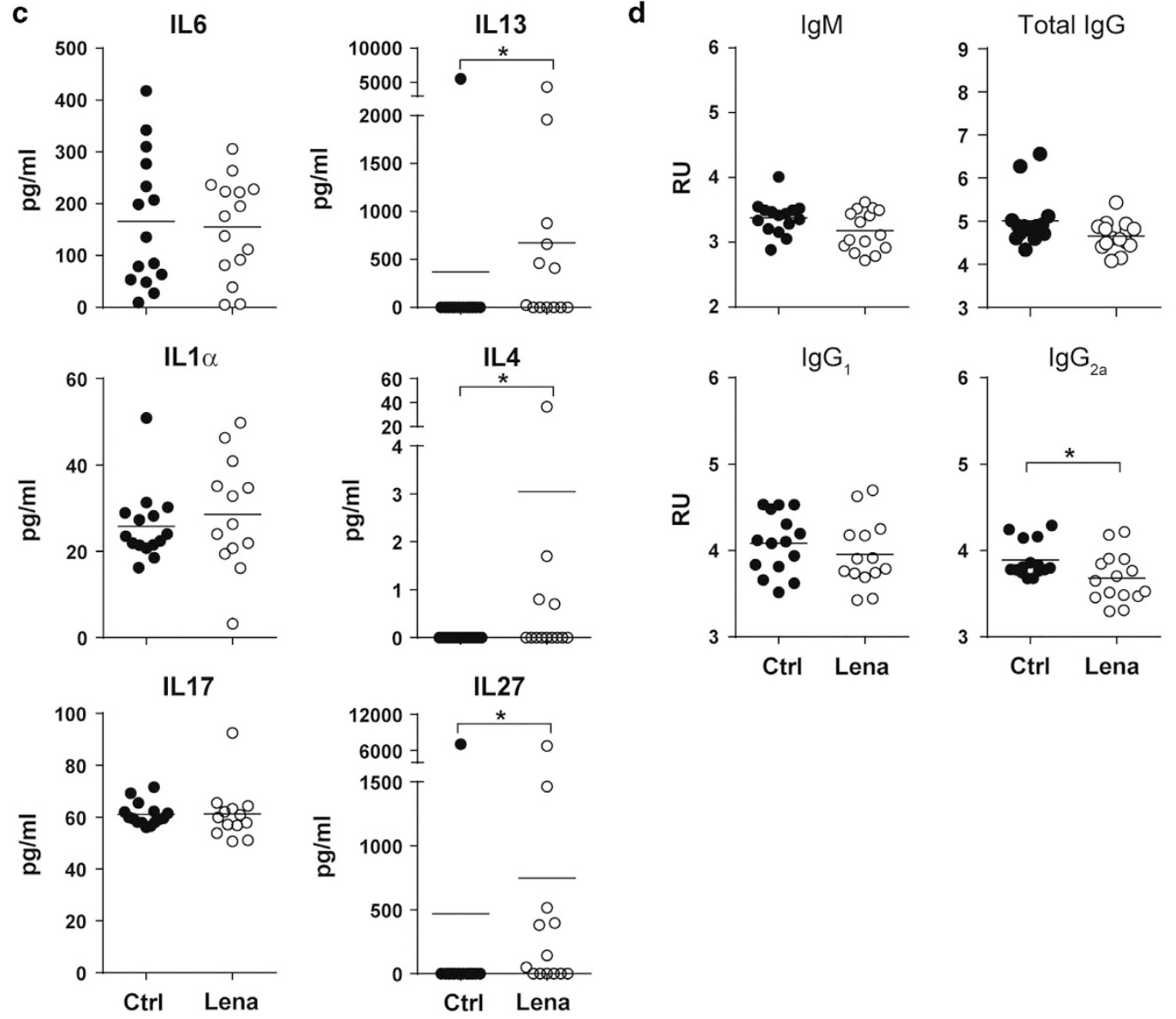

Figure 3 Lenalidomide treatment ameliorates established collagen-induced arthritis (CIA). Arthritis score (a) and number of affected paws (b) after therapeutic administration of Ienalidomide and pomalidomide in CIA mice. (c) Serum levels of IL6, IL1 $\alpha$, IL17, IL13, IL4 and IL27 in control- and lenalidomide-treated arthritic mice. (d) Levels of anti-Cll antibodies in sera from control and lenalidomide-treated mice. Data pooled from two independent experiments with eight mice per experimental group ( $n=16$ mice per group). Mean \pm s.e.m. of experimental groups is shown in $\mathbf{a}$ and $\mathbf{b}$, and values from individual mice and the mean of experimental groups are shown in $\mathbf{c}$ and $\mathbf{d}$. Statistical differences between groups were calculated as described in Materials and methods. ${ }^{*} P<0.05 ;{ }^{* *} P<0.01 ;{ }^{* *} P<0.001$. $\mathrm{RU}$, relative units.

\section{RESULTS}

Lenalidomide and pomalidomide protect against experimental IBD

We first investigated whether lenalidomide and pomalidomide have a therapeutic effect in two experimental models of acute colitis induced by intrarectal infusion of TNBS and by oral DSS administration, which display clinical, histopathological and immunological features similar to those of human Crohn's disease and ulcerative colitis. ${ }^{3,22}$ Inflammatory colitis was induced by intracolonic administration of TNBS and mice were treated i.p. for 3 days with lenalidomide or pomalidomide starting after TNBS instillation. TNBS-treated mice developed a 
a
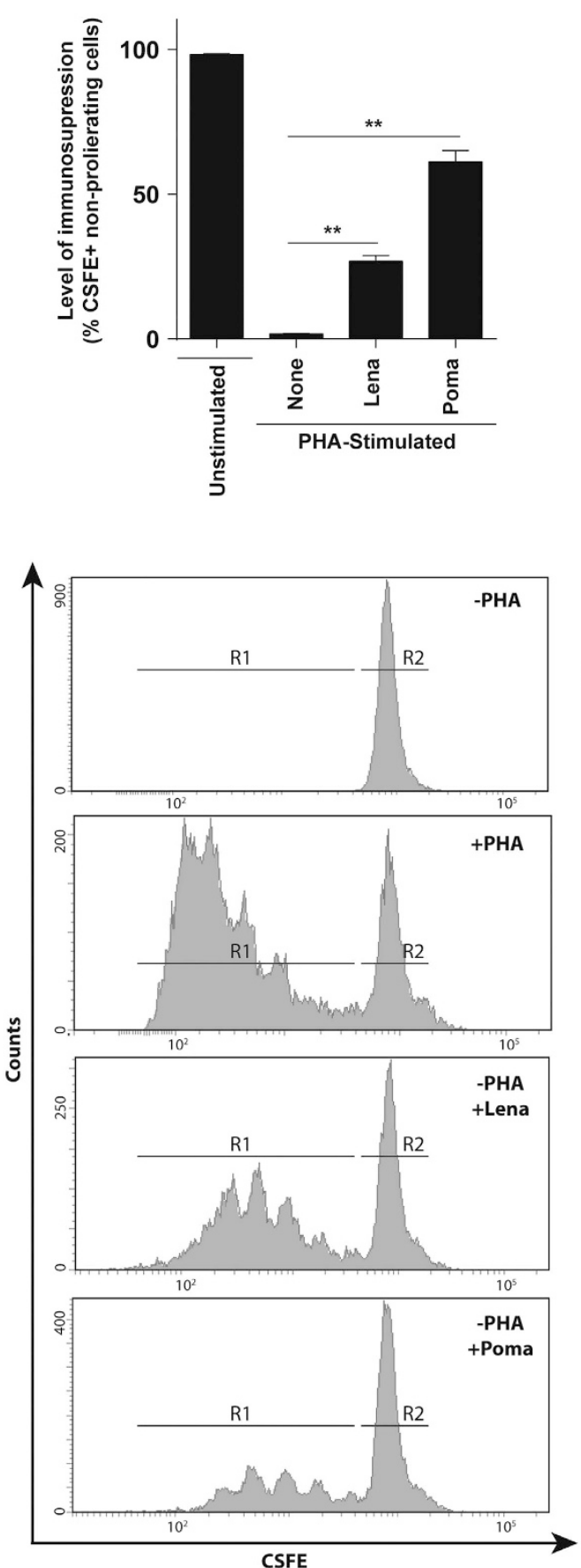

b
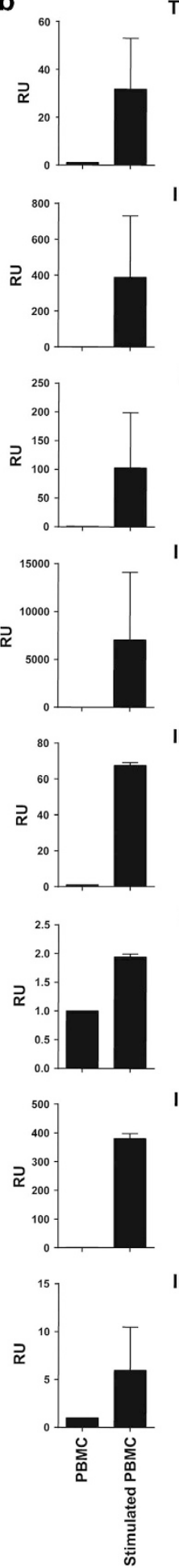

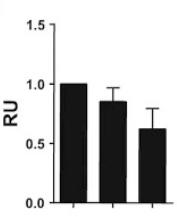

IL13

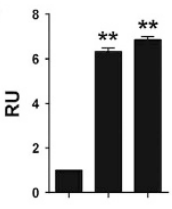

$\mathrm{TNF} \alpha$

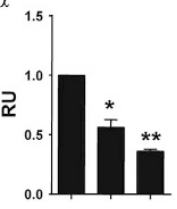

IL1 $\beta$

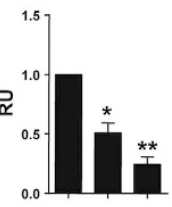

L6

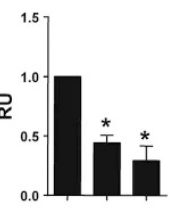

IFN $\gamma$

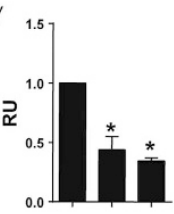

IL17
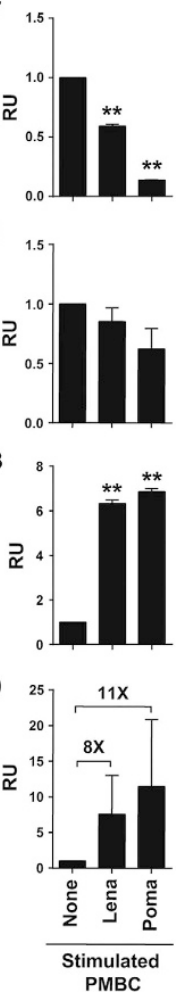

Figure 4 In vitro immunosuppressive and anti-inflammatory properties of lenaledomide and pomaledomide. (a) Carboxyfluorescein diacetate succinimidyl ester (CSFE)-labeled peripheral blood mononuclear cells (PBMCs) from healthy donors were stimulated or not with phytohemaglutinin (PHA). Then, PHA-stimulated PBMCs were treated with or without $10 \mu \mathrm{m}$ of lenalidomide or pomalidomide for 5 days. The number of cycling (CSFEmild/low) cells was determined by flow cytometry. Upper panel: percentage of non-proliferating cells expressed as mean \pm s.e.m. Lower panel: representative flow cytometry histograms of CSFE-labeled PBMCs. R1, proliferating cells; R2 nonproliferating cells. (b) Cytokine concentrations in cell culture supernatants were determined by Luminex Multiplex assays. PBMCs from healthy donors were stimulated or not with lipopolysaccharide (LPS or PHA for interferon gamma (IFN $\gamma$ ), IL17 and IL13). Stimulated PBMCs were treated with or without $10 \mu \mathrm{m}$ of lenalidomide or pomalidomide. Data are expressed as mean \pm s.e.m. Statistical differences between groups were calculated as described in Materials and methods. ${ }^{*} P<0.05 ;{ }^{*} P<0.01$; RU, relative units. 
severe acute illness characterized by substantial (20\%) and sustained body weight loss (Figure 1a, left panel), and by bloody diarrhea, rectal prolapse and pancolitis accompanied by extensive wasting syndrome (Figure 1b), that resulted in 100\% mortality within 7 days (Figure 1a, right panel). Macroscopic examination of colons revealed profound hyperemia, inflammation, necrosis and shortening (Figure 1c). Mice treated with lenalidomide were fully protected against IBD. Accordingly, lenalidomide-treated mice rapidly recovered $100 \%$ of their body weight loss within a 5-day time frame, with a significantly increased survival rate of $95 \%$ (Figure 1a). Furthermore, the wasting syndrome and the signs of colon inflammation were improved, leading to a significant decrease of the colon damage score, and mice regained a healthy appearance indistinguishable from ethanol-treated control mice (Figures $\mathrm{lb}$ and $\mathrm{c}$ ). Histological examination of the colons showed that treatment with lenalidomide significantly reduced the TNBS-induced transmural inflammation, depletion of mucin-producing goblet cells, epithelial ulceration, infiltration of inflammatory cells in the lamina propia and focal loss of crypts (Figure 1d). Pomalidomide treatment also ameliorated TNBS-induced colitis but its therapeutic effect was less potent than lenalidomide. Pomalidomide-treated mice recovered only $50 \%$ of their body weight loss and the survival rate reached only $40 \%$ (Figure 1a). This was reflected in a significant but modest improvement in colon inflammation and damage (Figures $1 \mathrm{~b}-\mathrm{d}$ ). These data demonstrate that lenalidomide fully protects mice against TNBS-induced IBD, whereas pomalidomide is less effective.

The therapeutic effect of lenalidomide was not exclusive to the TNBS-induced colitis model. Lenalidomide treatment was also very effective in ameliorating acute colitis induced with 3\% DSS (Figure 2). Lenalidomide significantly reduced the clinical activity, as shown by reduced body weight loss, improved stool consistency and decreased rectal bleeding (Figure 2a). Accordingly, lenalidomide ameliorated colon damage and reduced colon inflammation, revealed by an increased colon length/weight ratio (Figure 2b). Again, although treatment with pomalidomide showed some protective effect against the wasting disease and colon inflammation (Figure 2), it was less effective in comparison with lenalidomide.

\section{Lenalidomide treatment ameliorates established CIA}

The CIA mouse model is a faithful and well-established model that exhibits many clinical similarities to human RA, including synovitis, pannus formation, cartilage and bone erosion, fibrosis, and loss of joint function. ${ }^{20}$ Inflammatory arthritis was induced by collagen II immunization, and mice were treated daily with lenalidomide or pomalidomide starting on the day of arthritis onset. CIA mice developed symptomatic arthritis with at least two affected paws shortly after arthritis induction (Figures $3 \mathrm{a}$ and $\mathrm{b}$ ). Daily lenalidomide treatment in mice with established disease significantly reduced the severity of arthritis and the number of affected paws (Figures $3 a$ and $b$ ). By contrast, pomalidomide treatment was largely ineffective, and mice displayed little symptomatic improvement
(Figures 3a and b). Because IL6, IL1 $\alpha$, IL17 and anti-CII antibodies have a pathogenic role in the development of CIA, we measured their levels in the serum of arthritic mice treated or not with lenalidomide. In addition, we also analyzed the concentration in serum of other anti-inflammatory cytokines relevant in arthritis such as IL13, IL4 and IL27. The IL6, IL1 $\alpha$ and IL17 levels were unaffected by lenalidomide treatment (Figure 3c). Similarly, the levels of anti-CII IgM, IgG and $\operatorname{IgG}_{1}$ were similar between lenalidomide-treated and control groups (Figure 3d). However, the concentration of the antiinflammatory cytokines IL13, IL4 and IL27 was higher in lenalidomide-treated mice $\left(671 \pm 343\right.$ vs $370 \pm 370 \mathrm{pg} \mathrm{ml}^{-1}$, $P=0.01 ; 3.1 \pm 2$ vs $0 \pm 0 \mathrm{pg} \mathrm{ml}^{-1}, P=0.03$; and $748 \pm 514$ vs $468 \pm 468, P=0.01$, respectively). Importantly, specific anti-CII $\mathrm{IgG}_{2 \mathrm{a}}$ pathogenic antibodies were also moderately but significantly reduced in the lenalidomide-treated group $(4 \pm 0.05$ vs $3.6 \pm 0.07$ relative units, $P=0.04)$ (Figure $3 \mathrm{~d}$ ). Together, these findings show that lenalidomide, but not pomalidomide, exerts a therapeutic effect in the CIA model of RA.

\section{In vitro immunosuppressive and anti-inflammatory properties of lenaledomide and pomaledomide}

To address the possible mechanisms underlying the protective effect of IMiDs, we analyzed their immunosuppressive properties in vitro. Using CFSE dilution assays to monitor cell division, we found that both IMiDs suppressed the proliferation of PHA-stimulated human PBMCs (Figure 4a). The level of immunesuppression (measured as \% of IMiD-mediated non-proliferating PHA-stimulated lymphocytes) was $27 \pm 2 \%$ and $61 \pm 2 \%$ for lenalidomide and pomalidomide, respectively (Figure 4a). We then analyzed the cytokine profile in PHA- or lipopolysaccharide-activated human PBMCs treated or not with IMiDs. The production of master pro-inflammatory cytokines, such as TNF $\alpha$, IL1 $\beta$, IL6, IFN $\gamma$ and IL17 was significantly inhibited both by lenalidomide ( 2 -fold) and pomalidomide ( $>3$-fold), whereas IL8 levels were unchanged (Figure 4b). On the other hand, IMiDs increased the levels of anti-inflammatory cytokines such as IL13 ( 7-fold) and IL10 $(\sim 10$-fold) (Figure $4 \mathrm{~b})$. These data show that IMiDs exert immunosuppressive and anti-inflammatory activities both by inhibiting human lymphocyte proliferation and modulating the secretion of a broad range of cytokines toward an antiinflammatory profile, thus underpinning the therapeutic effects observed in experimental models of inflammatory disease.

\section{DISCUSSION}

Autoimmune/inflammatory diseases encompass a diverse group of debilitating inflammatory disorders prevalent in Western societies, and represent a major public health concern. Currently available therapies for autoimmune/inflammatory diseases are not entirely effective and are often non-specific. ${ }^{5,6}$ Biologic therapies for autoimmune/inflammatory diseases, such as those targeting specific molecules of the immune system, provide an alternative to the existing immunosuppressive treatments; however, the current limitations of such biologic 
therapies, including the inconvenience of intravenous administration, high cost and adverse events, prevent their widespread use as first-line treatments. ${ }^{23}$

Thalidomide is known to be an effective treatment option for Crohn's disease. ${ }^{24,25}$ Lienenlüke et al. ${ }^{26}$ showed a beneficial therapeutic effect of thalidomide in TNBS-induced colitis in rats through attenuation of colonic inflammation. Furthermore, clinical trials reported an effective action of thalidomide in steroid-dependent Crohn's disease. ${ }^{27-30}$ Despite its therapeutic promise, thalidomide is notoriously associated with severe birth defects, ${ }^{10}$ and was withdrawn from the market. ${ }^{11}$ Subsequently, thalidomide analogs (IMiDs) were developed and have emerged as potent and safe antineoplastic agents with immunomodulatory and antiangiogenic activities. ${ }^{12-14}$ The two first-in-class IMiDs with a favorable toxicity profile and increased efficacy are lenalidomide and pomalidomide, ${ }^{7,12}$ which are first-line treatments in several hematological malignancies. ${ }^{14,15}$ IMiDs are potent $\mathrm{TNF} \alpha$ inhibitors and block signaling through NFKB and COX-2, and have T-cell co-stimulatory properties. ${ }^{7,16-18,31,32}$ Mechanistically, IMiDs bind to cereblon, a substrate receptor of the CRL4 E3 ubiquitin ligase, altering its substrate specificity that results in breakdown of intrinsic downstream proteins such as Ikaros and Aiolos. ${ }^{33}$ IMiDs may therefore have a therapeutic window not only in neoplasia but also in autoimmune/inflammatory diseases.

Here we aimed to determine the potential therapeutic efficacy of lenalidomide and pomalidomide in wellestablished experimental models of IBD and arthritis. ${ }^{19-22}$ We found that lenalidomide produced a robust therapeutic effect in three experimental models of autoimmune/inflammatory disease. By contrast, pomalidomide administration resulted in a less-pronounced amelioration of symptoms. Nonetheless, both IMiDs exerted in vitro immunosuppressive effects on the in vitro proliferation of $\mathrm{T}$ lymphocytes and modulated the balance of cytokine production toward an antiinflammatory profile. Our results are in disagreement with Mansfield et al., ${ }^{34}$ who evaluated the efficacy and safety of 25 vs $5 \mathrm{mg}$ daily of lenalidomide as compared with placebo, in a randomized controlled clinical trial for moderate-to-severe Crohn's disease. In this study, the clinical remission rate of both treatment groups was not significantly different from that of the placebo group. Of note, patients enrolled in this study continued on their current corticosteroid treatment for the disease. Interestingly, a negative interaction of corticosteroids with the immunomodulatory effects of IMiDs has been extensively reported, thus explaining, at least in part, the poor efficacy of lenalidomide in this clinical trial. ${ }^{35,36}$ In addition, adverse events for lenalidomide at $25 \mathrm{mg}$ given in combination with corticosteroids resulted in a high rate of treatment discontinuation. However, we did not observed any adverse effects in animals with colitis treated with $50 \mathrm{mg}$ of lenalidomide alone. Therefore, more powerful and better designed clinical trials addressing the issue of dosing of lenalidomide as well as potential interaction with co-adjuvants are warranted. ${ }^{6}$
We also demonstrate a significant amelioration of symptoms in a RA mouse model treated with lenalidomide, but not with pomalidomide. In this respect, our results are in agreement with Oliver et al., who showed a therapeutic/immunosuppressive effect of thalidomide in a rat model of RA. ${ }^{37}$ Analogous to the IBD model, lenalidomide exerted an immunosuppressive effect on the proliferation of $\mathrm{T}$ lymphocytes and modulated the secretion of cytokines toward an anti-inflammatory profile. Of note, several immunosuppressive and biologic therapies have been shown to be effective for RA but not for Crohn's disease, and vice versa. ${ }^{38}$ A major strength of the present study is the therapeutic effect of lenalidomide in different experimental models of IBD and AR, which may have enormous implications for lenalidomide as a potential pan-treatment for a variety of autoimmune/inflammatory conditions. Lenaledomide, pomaledomide and thalidomide have been previously shown to exhibit distinct activity in $5 q^{-}$ syndrome $^{33}$ and in multiple myeloma. ${ }^{39}$ In fact, it was suggested that the levels of CRBN expression as well as the binding affinity of each IMiD to CRBN represent major determinants of IMiDs activity and resistance. Further mechanistic studies are required to decipher the differential mechanisms of action of lenaledomide vs pomaledomide in the in vivo models of autoimmune disease presented here. Nevertheless, more work is needed to unravel the precise mechanism(s) of action of lenalidomide and pomalidomide, which may underlie the differential potential of these IMiDs in vivo despite the robust inhibition of $\mathrm{T}$-cell proliferation and modulation of cytokine release towards an anti-inflammatory profile.

\section{CONFLICT OF INTEREST}

The authors declare no conflict of interest.

\section{ACKNOWLEDGEMENTS}

This work was supported by Health Canada (contract: H4080-144541) to PM and MR-M based on funding from Health Canada's Genomics Research and Development Initiative Phase VI (2014-2019) obtained by MR-M and JRL. PM also acknowledges the financial support from The Obra Social La Caixa-Fundaciò Josep Carreras and The Generalitat de Catalunya (SGR330). PM is investigator of the Spanish Cell Therapy cooperative network (TERCEL). We are indebted to Dominique Patry from Health Canada for his assistance with the Luminex experiments.

1 Cohen IR. Activation of benign autoimmunity as both tumor and autoimmune disease immunotherapy: a comprehensive review. J Autoimmun 2014; 54: 112-117.

2 Shi J, Chi S, Xue J, Yang J, Li F, Liu X. Emerging role and therapeutic implication of Wnt signaling pathways in autoimmune diseases. J Immunol Res 2016; 2016: 9392132.

3 Pizarro TT, Arseneau KO, Bamias G, Cominelli F. Mouse models for the study of Crohn's disease. Trends Mol Med 2003; 9: 218-222.

4 Williams RO, Inglis JJ, Simelyte E, Criado G, Sumariwalla PF. Analysing the effect of novel therapies on cytokine expression in experimental arthritis. Int J Exp Pathol 2005; 86: 267-278. 
5 Kasama T, Isozaki T, Takahashi R, Miwa Y. Clinical effects of tocilizumab on cytokines and immunological factors in patients with rheumatoid arthritis. Int Immunopharmacol 2016; 35: 301-306.

6 Srinivasan R, Akobeng AK. Thalidomide and thalidomide analogues for induction of remission in Crohn's disease. Cochrane Database Syst Rev 2009; 15;(2): CD007350.

7 Jelinek T, Kufova Z, Hajek R. Immunomodulatory drugs in AL amyloidosis. Crit Rev Oncol Hematol 2016; 99: 249-260.

8 Sharma D, Kwatra SG. Thalidomide for the treatment of chronic refractory pruritus. J Am Acad Dermatol 2016; 74: 363-369.

9 Song T, Ma X, Gu K, Yang Y, Yang L, Ma P et al. Thalidomide represses inflammatory response and reduces radiculopathic pain by inhibiting IRAK-1 and NF-kappaB/p38/JNK signaling. J Neuroimmunol 2016; 290: $1-8$.

10 Rajkumar SV. Thalidomide: tragic past and promising future. Mayo Clin Proc 2004; 79: 899-903.

11 Ghobrial IM, Rajkumar SV. Management of thalidomide toxicity. J Support Oncol 2003; 1: 194-205.

12 Quach H, Ritchie D, Stewart AK, Neeson P, Harrison S, Smyth MJ et al. Mechanism of action of immunomodulatory drugs (IMiDS) in multiple myeloma. Leukemia 2010; 24: 22-32

13 Sedlarikova L, Kubiczkova L, Sevcikova S, Hajek R. Mechanism of immunomodulatory drugs in multiple myeloma. Leuk Res 2012; 36: 1218-1224.

14 Touzeau C, Moreau P. Pomalidomide in the management of relapsed multiple myeloma. Future Oncol 2016; 12: 1975-1983.

15 Semeraro M, Vacchelli E, Eggermont A, Galon J, Zitvogel L, Kroemer G et al. Trial watch: lenalidomide-based immunochemotherapy. Oncoimmunology 2013; 2: e26494.

16 Davies FE, Raje N, Hideshima T, Lentzsch S, Young G, Tai YT et al. Thalidomide and immunomodulatory derivatives augment natural killer cell cytotoxicity in multiple myeloma. Blood 2001; 98: 210-216.

17 Payvandi F, Wu L, Haley M, Schafer PH, Zhang LH, Chen RS et al. Immunomodulatory drugs inhibit expression of cyclooxygenase-2 from TNF-alpha, IL-1beta, and LPS-stimulated human PBMC in a partially IL-10-dependent manner. Cell Immunol 2004; 230: 81-88.

18 Vallet S, Palumbo A, Raje N, Boccadoro M, Anderson KC. Thalidomide and lenalidomide: mechanism-based potential drug combinations. Leuk Lymphoma 2008; 49: 1238-1245.

19 Gonzalez MA, Gonzalez-Rey E, Rico L, Buscher D, Delgado M. Adipose-derived mesenchymal stem cells alleviate experimental colitis by inhibiting inflammatory and autoimmune responses. Gastroenterology 2009; 136: 978-989.

20 Gonzalo-Gil E, Perez-Lorenzo MJ, Galindo M, Diaz de la Guardia R Lopez-Millan B, Bueno C et al. Human embryonic stem cell-derived mesenchymal stromal cells ameliorate collagen-induced arthritis by inducing host-derived indoleamine 2,3 dioxygenase. Arthritis Res Ther 2015; 18: 77

21 Gonzalez-Rey E, Anderson P, Gonzalez MA, Rico L, Buscher D, Delgado M. Human adult stem cells derived from adipose tissue protect against experimental colitis and sepsis. Gut 2009; 58: 929-939.

22 Sanchez L, Gutierrez-Aranda I, Ligero G, Rubio R, Munoz-Lopez M, Garcia-Perez JL et al. Enrichment of human ESC-derived multipotent mesenchymal stem cells with immunosuppressive and anti-inflammatory properties capable to protect against experimental inflammatory bowel disease. Stem Cells 2011; 29: 251-262.

23 Rosman Z, Shoenfeld Y, Zandman-Goddard G. Biologic therapy for autoimmune diseases: an update. BMC Med 2013; 11: 88.

24 Ginsburg PM, Dassopoulos T, Ehrenpreis ED. Thalidomide treatment for refractory Crohn's disease: a review of the history, pharmacological mechanisms and clinical literature. Ann Med 2001; 33: 516-525.

25 Fakhoury M, Coussa-Charley M, Al-Salami H, Kahouli I, Prakash S Use of artificial cell microcapsule containing thalidomide for treating
TNBS-induced Crohn's disease in mice. Curr Drug Deliv 2014; 11: 146-153.

26 Lienenluke B, Stojanovic T, Fiebig T, Fayyazi A, Germann T, Hecker M. Thalidomide impairment of trinitrobenzene sulphonic acid-induced colitis in the rat - role of endothelial cell-leukocyte interaction. $\mathrm{Br} J$ Pharmacol 2001; 133: 1414-1423.

27 Ehrenpreis ED, Kane SV, Cohen LB, Cohen RD, Hanauer SB. Thalidomide therapy for patients with refractory Crohn's disease: an open-label trial. Gastroenterology 1999; 117: 1271-1277.

28 Vasiliauskas EA, Kam LY, Abreu-Martin MT, Hassard PV, Papadakis KA, Yang $\mathrm{H}$ et al. An open-label pilot study of low-dose thalidomide in chronically active, steroid-dependent Crohn's disease. Gastroenterology 1999; 117: 1278-1287.

29 Plamondon S, Ng SC, Kamm MA. Thalidomide in luminal and fistulizing Crohn's disease resistant to standard therapies. Aliment Pharmacol Ther 2007; 25: 557-567.

30 Lazzerini M, Martelossi S, Marchetti F, Scabar A, Bradaschia F, Ronfani L et al. Efficacy and safety of thalidomide in children and young adults with intractable inflammatory bowel disease: long-term results. Aliment Pharmacol Ther 2007; 25: 419-427.

31 Geitz H, Handt S, Zwingenberger K. Thalidomide selectively modulates the density of cell surface molecules involved in the adhesion cascade. Immunopharmacology 1996; 31: 213-221.

32 Jin SH, Kim TI, Yang KM, Kim WH. Thalidomide destabilizes cyclooxygenase-2 mRNA by inhibiting p38 mitogen-activated protein kinase and cytoplasmic shuttling of HuR. Eur J Pharmacol 2007; 558 : 14-20.

33 Ito T, Handa H. Cereblon and its downstream substrates as molecular targets of immunomodulatory drugs. Int J Hematol 2016; 104: 293-299.

34 Mansfield JC, Parkes M, Hawthorne AB, Forbes A, Probert CS, Perowne RC et al. A randomized, double-blind, placebo-controlled trial of lenalidomide in the treatment of moderately severe active Crohn's disease. Aliment Pharmacol Ther 2007; 26: 421-430.

35 Hsu AK, Quach H, Tai T, Prince HM, Harrison SJ, Trapani JA et al. The immunostimulatory effect of lenalidomide on NK-cell function is profoundly inhibited by concurrent dexamethasone therapy. Blood 2011; 117: 1605-1613.

36 Gandhi AK, Kang J, Capone L, Parton A, Wu L, Zhang LH et al. Dexamethasone synergizes with lenalidomide to inhibit multiple myeloma tumor growth, but reduces lenalidomide-induced immunomodulation of T and NK cell function. Curr Cancer Drug Targets 2010; 10: 155-167.

37 Oliver SJ, Cheng TP, Banquerigo ML, Brahn E. The effect of thalidomide and 2 analogs on collagen induced arthritis. J Rheumatol 1998; 25: 964-969.

38 Sandborn WJ, Hanauer SB, Katz S, Safdi M, Wolf DG, Baerg RD et al. Etanercept for active Crohn's disease: a randomized, double-blind, placebo-controlled trial. Gastroenterology 2001; 121: 1088-1094.

39 Zhu YX, Kortuem KM, Stewart AK. Molecular mechanism of action of immune-modulatory drugs thalidomide, lenalidomide and pomalidomide in multiple myeloma. Leuk Lymphoma 2013; 54: 683-687.

This work is licensed under a Creative Commons Attribution-NonCommercial-NoDerivs 4.0 InterAttribution-NonCommercial-NoDervs 4.0 Interthis article are included in the article's Creative Commons license, unless indicated otherwise in the credit line; if the material is not included under the Creative Commons license, users will need to obtain permission from the license holder to reproduce the material. To view a copy of this license, visit http://creativecommons.org/licenses/by-nc-nd/4.0/ 